\title{
Template Co-update in Multimodal Biometric Systems
}

\author{
Fabio Roli ${ }^{1}$, Luca Didaci ${ }^{2}$, and Gian Luca Marcialis ${ }^{1}$ \\ ${ }^{1}$ University of Cagliari - Department of Electrical and Electronic Engineering \\ Piazza d'Armi - 09123 Cagliari (Italy) \\ ${ }^{2}$ University of Cagliari - Department of Pedagogical and Philosophical Sciences \\ Via Is Mirrionis, 1 - 09123 Cagliari (Italy) \\ \{roli, luca.didaci, marcialis\} @diee.unica.it
}

\begin{abstract}
Performances of biometric recognition systems can degrade quickly when the input biometric traits exhibit substantial variations compared to the templates collected during the enrolment stage of users. This issue can be addressed using template update methods. In this paper, a novel template update method based on the concept of biometric co-training is presented. In multimodal biometric systems, this method allows co-updating the template galleries of different biometrics, realizing a co-training process of biometric experts which allows updating templates more quickly and effectively. Reported results provide a first experimental evidence of the effectiveness of the proposed template update method.
\end{abstract}

\section{Introduction}

As pointed out clearly by Uludag et al. [1], in real operational scenarios, input biometric data can exhibit substantial variations compared to the templates collected during the enrolment stage of users. In other words, we can have a large intra-class variability, due to changes of the environment conditions (e.g., illumination changes), aging of the biometric traits, variations of the interaction between the sensor and the individual (e.g., variations of the person pose), etc. This large intra-class variability can make the templates acquired during the enrolment session poorly representative of the biometric data to be recognized, so resulting in poor recognition performances. For example, Tan et al. pointed out, by experiments with the ORL face data base [2], that the performance of an eigenface-based face recogniser drops quickly when the enrolled templates become poorly representative. The authors reported similar results for the AR face data base [3]. Template update methods have been proposed to address this issue. In fingerprint recognition, Uludag et al. proposed two methods which can be used to update templates when new fingerprint impressions, labeled with the user's identity, become available [1]. One of the methods is based on a clustering strategy to choose a template set that best represents the intra-class variations, whilst the other selects templates that exhibit maximum similarity with the rest of the impressions. Both the methods work off-line (i.e., template updating is performed after a new batch of labelled data has been acquired) and are supervised (i.e., a supervisor has to label the new biometric data to be used for template updating). Some "semi-supervised" template updating methods have been also 
proposed. With the term semi-supervised, we indicate those methods that update templates using both the initial set of labelled templates and a set of unlabelled data acquired during the on-line operation of the biometric system. Jiang and Ser [ 4], Ryu et al. [5], proposed some on-line and semi-supervised methods which update templates by a fusion process with impressions acquired on-line which are recognized as genuine with high reliability. Liu et al. proposed an on-line semi-supervised algorithm to update incrementally the eigenspace of a PCA-based face recognition system by exploiting unlabelled data acquired during the system's operation [6]. Roli and Marcialis developed an off-line semi-supervised method, using the learning method named "self-training", to update incrementally the templates and the eigenspace of a PCA-based face recognition system [3].

To our knowledge, so far template update methods have focused on unimodal biometric systems, and no method tailored to multimodal systems has been proposed. In addition, the performance of unimodal, semi-supervised, template update methods depends on two contrasting factors: the importance of updating templates using "difficult", but informative, new biometric data, and the difficulty to recognize correctly new input data that exhibit substantial variations compared to the current templates. It is easy to see that a template update method which exploits only high quality and easy to recognize input data may not be able to account for the variations of an user's biometric.

In this paper, a novel template update method tailored to multimodal biometric systems is proposed. This method exploits the concept of biometric co-training that the authors introduced in [7], and it is based on the semi-supervised learning method proposed by Blum and Mitchell [8]. In multimodal biometric systems, this method allows co-updating the template galleries of different biometrics, realizing a cotraining process of biometric experts which allows updating templates also using "difficult", but informative, input data. Results reported in Section 4 provide a first experimental evidence of the effectiveness of the proposed template update method.

\section{Template Co-update and Biometric Co-training}

The template co-update method we developed was inspired by the semi-supervised learning method named co-training [8], and, therefore, it can regarded as a biometric co-training algorithm where two multimodal biometric experts co-train each other in order to update their template galleries. The key idea behind biometric co-training is a generalization to the problem of template update of the basic idea behind multi-modal biometrics [7]. In fact, the "complementary" performances of biometric recognizers using distinct biometric traits, for example, face and fingerprints, are one of the fundamental motivations for multi-modal biometrics [9]. Intuitively, each recognizer is expected to assign correct labels to certain input data which are difficult for the other. So far this idea was the basis for the design of multi-modal systems, but such idea has never been exploited in a learning context to implement a template update method. On the other hand, the complementary performances of two biometric recognizers can be indeed exploited in a semi-supervised learning context, by allowing the recognizers to co-train each other. A co-training approach to semisupervised learning was proposed by Blum and Mitchell in 1998 [8]. Here we 
illustrate the basic idea behind the original co-training approach of Blum and Mitchell with a multi-modal biometric recognition example, which also allows us to explain how this semi-supervised learning method could be used to implement a template update method. Let us suppose that we have a multimodal biometric system made up of a face and a fingerprints recognition system, and a small set $D_{l}$ of biometric data, labelled with the users' identities, acquired during the enrolment session. According to the Blum and Mitchell co-training algorithm, these two recognisers should be trained with the set $D_{l}$, and it is assumed that they will exhibit a low, but better than random, accuracy. In our biometric example, the set $D_{l}$ should be used to create the initial template galleries, and, eventually, to initialize other parameters of the recognition system (e.g., computing the eigenspace in the case of a PCA-based face recogniser). During the on-line operation of the biometric system, a batch $D_{u}$ (usually, much larger than $D_{l}$ ) of unlabelled data can be acquired (this data set is obtained simply by disregarding the labels assigned by the recognition system). The co-training algorithm assumes that each recognizer is applied to such unlabeled set $D_{u}$. For each recogniser, the unlabelled examples that are classified with the highest confidence are added to the training set $D_{l}$, so that the two recognisers contribute to increase the data set. Both the recognisers are re-trained with this augmented data set (e.g., by updating the face and fingerprint template galleries), and the process is repeated a specified number of times. Intuitively, co-training is expected to work because each recogniser may assign correct labels to certain examples, whilst it may be difficult for the other recogniser to do so. Therefore, each recogniser can increase the training set with examples which are very informative for the other recogniser. In few words, the two recognisers are expected to co-train each other. When the co-training process finishes, the two resulting recognisers can be combined by the product of their outputs.

It is worth noting that a fundamental assumption of the Blum and Mitchell cotraining algorithm is that patterns are represented with two "redundantly sufficient" feature sets. This assumption means that the two feature sets should be conditionally independent, so that the examples which are classified with high confidence by one of the two recognisers are i.i.d. samples for the other, and both the feature sets should be sufficient to design an optimal recognition algorithm if we have enough labelled data. In multi-modal biometrics, using, for example, face and fingerprint data, this assumption is likely to be satisfied, at least at the extent which justifies the use of cotraining for practical purposes.

Figure 1 illustrates the use of biometric co-training to co-update face and fingerprints galleries. A given bi-modal, face and fingerprint, input data could be difficult to classify correctly for the face recogniser using the available templates (e.g., due to an illumination change), while the fingerprint recogniser could be able to do easily because the user provided a good fingerprint impression. In Figure 1, this is the case of the first couple of face-fingerprint images on the right of the initial templates. In this case, the fingerprint recogniser could train the face recogniser, that is, the face template gallery could be updated using this difficult (and, therefore, "informative") face image which was acquired jointly with an easy fingerprint image. This update of the face template gallery would not be possible without co-training (because we are supposing that the input face image cannot be recognised correctly with the current templates in the gallery), or this would take much more time (i.e., one 


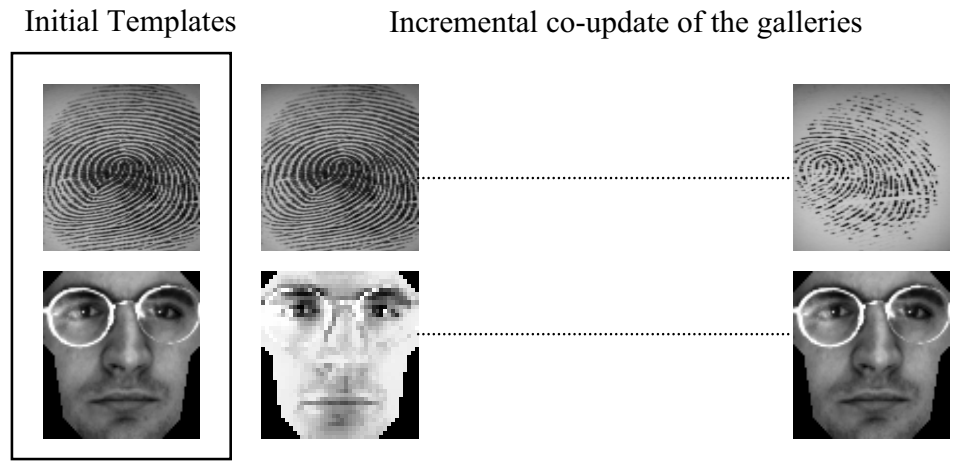

Fig. 1. An illustrative example of the use of biometric co-training to co-update face and fingerprints galleries. The first couple of images on the left are the initial face and fingerprint templates. Two examples of co-update of the galleries are shown on the right of the templates. These examples highlight how a difficult (and, therefore, "informative") biometric template can be added to a gallery, supposed that this input data comes in with a sample of the other biometrics which can be easily recognized using the initial template.

should wait for an incremental update of the gallery which makes possible to recognise correctly this difficult face input). The fingerprint recogniser can train the face recogniser in a similar way (see the case of the second couple, on the right of the initial templates, of input face-fingerprint images in Figure 1), so realizing a cotraining process which allow updating templates more quickly and effectively.

\section{Co-updating of Face and Fingerprint Recognition Systems}

In order to investigate the practical use of a template co-update method based on the above biometric co-training concept, we first implemented a simple multi-modal identification system made up of a PCA-based face recognizer and a fingerprint recognizer using the "String" matching algorithm ("String" is a matching algorithm based on minutiae points). We used the standard versions of these two recognition algorithms $[10,11]$. Then we implemented the template co-update algorithm in Figure 2 to update automatically the template galleries of these two recognizers. It is worth noting that our co-update algorithm also updates the eigenspace of the PCA-based face recognizer, as we experimented that this improves the overall performance of the multimodal system w.r.t. updating only the template galleries. The main steps of the co-updating algorithm we implemented are summarized in Figure 2. After the enrolment session, the set $D_{l}$, containing the face and fingerprint images acquired, labeled with the user's identity, is used to train the PCA-based face recognizer (i.e., the PCA transform is computed and the initial templates are created) and to create the initial fingerprint templates. Then, during the on-line operation, an unlabelled batch of data $D_{u}$ is collected over a given period of time, simply by disregarding the labels assigned by the recognition system. This set $D_{u}$ is used for the template co-update process as follows (Figure 2). According to the standard co-training approach, for each iteration of the algorithm, two sets $D^{l}$ and $D^{2}$ of unlabelled images recognized 
with highest confidence (i.e., the images nearest to the class templates) by the face and fingerprint recognizer, respectively, are added to the training set $D_{l}$, and this augmented training set is used to update the eigenspace, and the face and fingerprint templates. In our algorithm the update of fingerprint templates is performed simply by adding the unlabelled images recognized with highest confidence (i.e., the images contained in the set $D^{2}$ ) to the user's gallery. This simple method could not be used in a practical scenario, as the size of the template gallery could become too large (we used it in our experiments only to assess the proposed approach). However, template selection methods, as the ones a proposed in [1], can be used to reduce the size of the gallery during the template co-update process. In the recognition phase of our system, the input fingerprint image is matched against all the templates of the gallery, and the final matching score is computed as average of the individual scores. For each identity, the face template is simply the "mean" face, so template updating is very simple. However, more sophisticated methods, based on clustering, could be used for updating the face templates [1].

Finally, one should note that we are assuming that the template co-updating process is performed either when the system is not operating (e.g., during the night) or using a separate processing unit which allows carrying out co-updating in parallel with the recognition stage.

\section{Experimental Results}

The goal of our experiments was to evaluate the capability of the proposed coupdating algorithm to exploit a batch of unlabelled images, collected during a given session of the system operation, in order to update the face and fingerprint templates galleries, and the eigenspace of the PCA-based face recognizer. To this end, we carried out experiments with the AR and the FVC-2002 DB2 data sets on an identification task $[12,13]$. The $\mathrm{AR}$ data set contains frontal view faces with different facial expressions, illumination conditions, and occlusions (sun glasses and scarf). Each person participated in two acquisition sessions, separated by two weeks time. Each session is made up of seven images per person. We selected 100 subjects (50 males and 50 females), and manually cropped face images and, after histogram stretching and equalization, resized them at 40x40 pixels. The FVC-2002 DB2 data set is made up of 800 fingerprints images, acquired with an optical sensor, belonging to 100 subjects. For each individual, eight fingerprint impressions have been acquired. We coupled the two data bases in two different ways in order to create two "chimerical" multi-modal data sets for our experiments. One of the chimerical data sets was created by selecting, for each user, one face image and one fingerprint impression as training set $D_{l}$, that is, as initial templates (in particular, the face templates were selected from the first acquisition session of the AR data base), and using the remaining seven fingerprint impressions and seven face images of the second AR session as unlabelled data set $D_{u}$. The other chimerical data set was created simply reversing the use of the AR images, that is, selecting the training data from the second session images and the unlabelled data from the first session images. In addition, for each data set, we performed two trials using either the face or the 
In the enrolment session, collect a set $D_{l}$ of labelled face and fingerprint images. A couple of face and fingerprint images is acquired for each user.

Compute the PCA transform and create the face templates using the set $D_{l}$

Create the fingerprint templates using the set $D_{l}$

During the on-line system operation, collect an unlabelled set $D_{u}$.

$* *$ Co-update algorithm**

Loop for $N$ iterations:

Assign (pseudo)identity labels to a subset $D^{l}$ of images in $D_{u}$ recognized with high confidence by the face recogniser

Assign (pseudo)identity labels to a subset $D^{2}$ of images in $D_{u}$ recognized with high confidence by the fingerprint recogniser

Increase the training set $D_{l} \leftarrow D^{1} \cup D^{2}$

Update PCA transform using the augmented labelled set $D_{l}$

Update face templates using the augmented labelled set $D_{l}$

Update fingerprint templates using the augmented labelled set $D_{l}$

Fig. 2. Co-updating algorithm of a PCA-based face recogniser and a fingerprint matcher using the "String" algorithm. The main steps of the algorithm are shown.

fingerprint recogniser as first algorithm, in the loop of the co-updating algorithm (Figure 2), which assigns (pseudo)labels to unlabelled data. In fact, even if, theoretically speaking, this order should not affect co-updating performances, we verified that it can do in this biometric application. In summary, the results which are going to report are the average of the results obtained with four trials (two data sets and two trials for each data set).

Figure 3 shows the percentage accuracy on the unlabelled data set $D_{u}$ averaged on four trials. For this experiment, we point out that the initial galleries (before the template co-update process) contained only one face and fingerprint template per person. Performances are shown as function of the number of unlabelled data used during the iterations of the co-updating algorithm. Around one-hundred pseudolabeled data were added to the training set during every iteration. Looking the two curves labelled "Co-updating-Fingerprint" and "Co-updating-Face" in Figure 3 one notes immediately the large difference of performance between the fingerprint and face recognizer at the beginning of the co-updating process, when no unlabeled data has been still used (90\% vs. $48 \%$ of accuracy). This large difference is due to the use of a single template per person and the characteristics of the two data sets. For the AR face data set, the large differences between first and second session images make a single template per person poorly representative. Differently, for the FVC-2002 DB2 fingerprint data set, a single template per person is quite representative of the remaining seven fingerprint impressions, as the results of the past FVC-2002 competion pointed out [13]. It is worth noting that such a performance unbalance between a face and a fingerprint recogniser is a realistic scenario. In fact, large 
differences of performances have been reported in many works [9], and they could be indeed exhibited when a multi-modal system is created by adding a new face recognition module to a previously installed, and well trained, fingerprint recognition system. In this case, the face recognition module could initially exhibit performances much lower than the ones of the fingerprint module whose templates have been (re)updated by supervised (re)enrolment sessions over the time. It should be also noted that co-training can offer a solution to this kind of practical cases. In fact, the face recognizer, newly installed, could be co-trained by the fingerprint recognizer. Other scenarios, where performances are more balanced, are obviously possible, and we are investigating by experiments such cases.

Figure 3 shows clearly that the fingerprint and the face recogniser co-train each other, that is, their accuracies increase substantially with the number of unlabelled data exploited by co-training. As it could be expected, the face recogniser, whose initial templates were poorly representative, gets the greatest benefits from the coupdating process. It is also interesting to note that the combination of the two recognisers by product of their matching scores further increases the performance. From an application viewpoint, this results points out that the co-training process can allow to improve the recognition results previously achieved on a batch of input data. For example, in a person identification scenario such a system re-training could allow improving the identification results stored in the data base the day before. We assessed by experiments that co-training can also improve recognition accuracy on novel input data, acquired after the co-training process with the unlabeled set $D_{u}$. The benefits of co-training were also assessed in terms of the so called rank-order curves. For the sake of brevity, we do no report these results.

To investigate the practical operation of the proposed template co-updating algorithm, we analysed how the galleries of users' templates were updated and increased. We report this analysis only for the face galleries, as it is easier to understand the operation by the visual analysis and comparison of face galleries than analysing fingerprint galleries. We also compared the galleries created by our template co-update method with the ones created by the self-update method of face templates we proposed in [3]. Such method selects the unlabelled data to be used for template updating in a way similar to the one proposed for fingerprint template updating in $[4,5]$. Face templates are updated using the unlabelled images recognized with highest confidence (i.e., the images nearest to the current class templates). This comparison should help the reader to understand better the advantages of co-updating.

Figure 4 depicts an examples of the update of users' galleries by co-updating and self-updating. For each gallery, the first image on the left is the initial training image used as face template. The remaining images are the unlabelled images which were pseudo labelled and added to the galleries during the iterations of co-updating and self-updating. In Figure 4, if one compare the gallery depicted in the first row, created by co-updating, with the second row gallery, created by self-updating, it is easy note that our co-updating algorithm allows updating the gallery with "difficult" (and, therefore, "informative") face images quicker than self-updating. For example, the second image of the first row is a difficult image w.r.t. the initial template (illumination changed substantially); and, in fact, self-updating added this image only at the fourth iteration. 


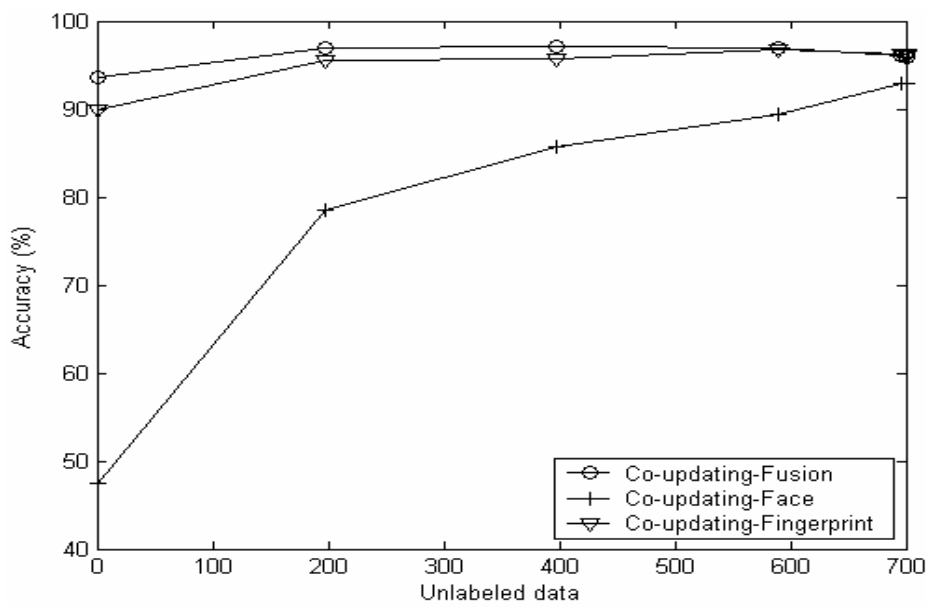

Fig. 3. Average accuracy on the unlabelled data set as function of the number of unlabelled data used in the co-updating algorithm of Figure 2. The curves "Co-updating-Fingerprint" and "Coupdating-Face" refer to the co-trained fingerprint and face recognition algorithms, respectively. The curve "Co-updating-Fusion" refers to the combination of the two algorithms by the product of their matching scores.

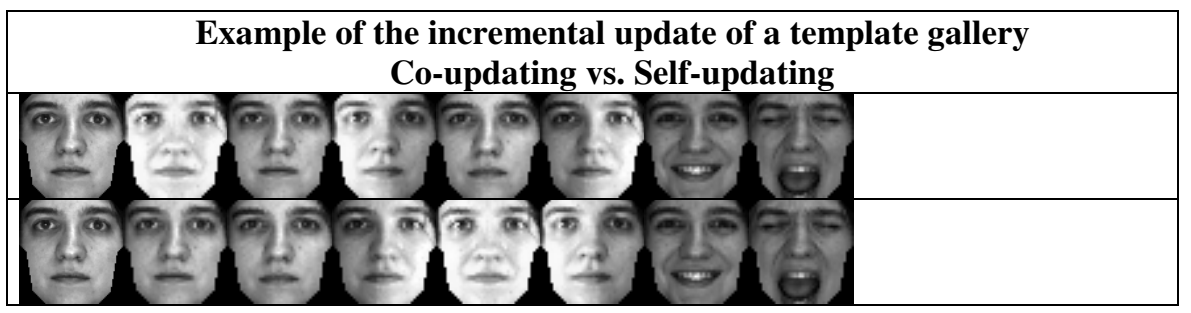

Fig. 4. Example of the incremental update of a face gallery. A comparison between the galleries created by co-updating and self-updating is shown. For all the galleries, the first image on the left is the initial training image used as face template. First row: gallery created by co-updating; second row: gallery created by self-updating for the same user.

\section{Conclusions}

In this paper, we presented a novel template update method. Our method exploits the concept of biometric co-training that can be regarded as an application of the semisupervised learning method proposed by Blum and Mitchell. In multimodal biometric systems, this method allows co-updating the template galleries exploiting also"difficult", but informative, input data. Results reported in Section 4 provide a first experimental evidence of the effectiveness of the proposed template update method.

It is worth noting that, in a multi-modal biometric system, various system configurations are possible, depending on the application, the number of sensors, biometric traits, recognition algorithms, etc.. We believe that Investigating the use of 
co-training for different configurations of a multi-modal biometric system is an interesting issue for future research. Finally, we point out that co-training can be also used in biometric systems using more than two recognisers (e.g., more than two biometric traits), supposed that this ensemble of recognisers satisfy the assumptions of co-training algorithm on the feature sets. It should be noted, however, that cotraining of an ensemble of classifiers is still a matter of on-going research, as cotraining has always been used with just two classifiers.

\section{References}

[1] Uludag, U., Ross, A., Jain, A.K.: Biometric template selection and update: a case study in fingerprints. Pattern Recognition 37(7), 1533-1542 (2004)

[2] Tan, X., Chen, S., Zhou, Z.-H., Zhang, F.: Face recognition from a single image per person: a survey. Pattern Recognition 39(9), 1725-1745 (2006)

[3] Roli, F., Marcialis, G.L.: Semi-supervised PCA-based face recognition using selftraining. In: Yeung, D.-Y., Kwok, J.T., Fred, A., Roli, F., de Ridder, D. (eds.) Structural, Syntactic, and Statistical Pattern Recognition. LNCS, vol. 4109, pp. 560-568. Springer, Heidelberg (2006)

[4] Jiang, X., Ser, W.: Online Fingerprint Template Improvement. IEEE Trans. PAMI 24(8), 1121-1126 (2002)

[5] Ryu, C., Hakil, K., Jain, A.K.: Template adaptation based fingerprint verification. In: Proc. of International Conference on Pattern Recognition (ICPR), Hong Kong, August, vol. 4, pp. 582-585 (2006)

[6] Liu, X., Chen, T., Thornton, S.M. (2003) Eigenspace updating for non-stationary process and its application to face recognition. Pattern Recognition, 1945-1959 (2003)

[7] Roli, F., Didaci, L., Marcialis, G.L.: Adaptive biometric systems that can improve with use, Advances in Biometrics. In: Ratha, N., Govindaraju, V. (eds.) Sensors, Systems and Algorithms (2007) (in press)

[8] Blum, A., Mitchell, T.: Combining labeled and unlabeled data with co-training. In: Proc. of the Workshop on Computational Learning Theory, pp. 92-100 (1998)

[9] Ross, A., Nandakumar, K., Jain, A.K.: Handbook of Multibiometrics. Springer, Heidelberg (2006)

[10] Turk, M., Pentland, A.: Eigenfaces for Face Recognition. Journal of Cognitive Neuroscience 3(1), 71-86 (1991)

[11] Jain, A.K., Hong, L., Bolle, R.: On-line Fingerprint Verification. IEEE Transactions on Pattern Analysis and Machine Intelligence 19(4), 302-314 (1997)

[12] Martinez, A., Benavente, R.: The AR Face Database. CVC Technical Report \#24 (June 1998)

[13] Maio, D., Maltoni, D., Cappelli, R., Wayman, J.L., Jain, A.K.: FVC2002: Second Fingerprint Verification Competition. In: Proceedings 16th International Conference on Pattern Recognition (ICPR2002), Québec City, vol. 3, pp. 811-814 (2002) 